\title{
Tides in asynchronous binary systems
}

\author{
O. Toledano ${ }^{1}$, E. Moreno ${ }^{2}$, G. Koenigsberger ${ }^{1}$, R. Detmers ${ }^{3}$, and N. Langer ${ }^{3}$ \\ 1 Instituto de Ciencias Físicas, Universidad Nacional Autonoma de México, Apdo. Postal 48-3, Cuernavaca, Mor. 62251, Mexico \\ e-mail: [oswaldo;gloria]@ce.fis.unam.mx \\ 2 Instituto de Astronomía, Universidad Nacional Autonoma de México, Apdo. Postal 70-264, D.F. 04510, Mexico \\ e-mail: edmundo@astroscu. unam.mx \\ 3 Sterrenkundig Instituut, Universiteit Utrecht, Postbus 80.000, Utrecht, The Netherlands \\ e-mail: [R.G.Detmers; N.Langer]@astro.uu.nl
}

Received 8 June 2006 / Accepted 8 October 2006

\begin{abstract}
Context. Stellar oscillations are excited in non-synchronously rotating stars in binary systems due to the tidal forces. Tangential components of the tides can drive a shear flow which behaves as a differentially forced rotating structure in a stratified outer medium. Aims. The aims of this paper are to show that our single-layer approximation for the calculation of the forced oscillations yields results that are consistent with the predictions for the synchronization timescales in circular orbits, $\tau_{\mathrm{sync}} \sim a^{6}$, thus providing a simplified means of computing the energy dissipation rates, $\dot{E}$. Furthermore, by calibrating our model results to fit the relationship between synchronization timescales and orbital separation, we are able to constrain the value of the kinematical viscosity parameter, $v$.

Methods. We compute the values of $\dot{E}$ for a set of $5 M_{\odot}+4 M_{\odot}$ model binary systems with different orbital separations, $a$, and use these to estimate the synchronization timescales.

Results. The resulting $\tau_{\text {synch }}$ vs. $a$ relation is comparable to that of Zahn (1977, A\&A, 57, 383) for convective envelopes, providing a calibration method for the values of $v$. For the $4+5 M_{\odot}$ binary modeled in this paper, $v$ is in the range $0.0015-0.0043 R_{\odot}^{2} /$ day for orbital periods in the range $2.5-25 \mathrm{~d}$. In addition, $\dot{E}$ is found to decrease by $\sim 2$ orders of magnitude as synchronization is approached, implying that binary systems may approach synchronization relatively quickly but that it takes a much longer timescale to actually attain this condition.

Conclusions. The relevance of these results is threefold: 1) our model allows an estimate for the numerical value of $v$ under arbitrary conditions in the binary system; 2) it can be used to calculate the energy dissipation rates throughout the orbital cycle for any value of eccentricity and stellar rotational velocity; and 3) it provides values of the tangential component of the velocity perturbation at any time throughout the orbit and predicts the location on the stellar surface where the largest shear instabilities may be occurring. We suggest that one of the possible implication of the asymmetric distribution of $\dot{E}$ over the stellar surface is the generation of localized regions of enhanced surface activity.
\end{abstract}

Key words. stars: oscillations - stars: rotation - stars: binaries: general

\section{Introduction}

A binary system is said to be in equilibrium when the orbit is circular, the stellar rotation period and the orbital period are synchronized and the spin axes of both stars are aligned and perpendicular to the orbital plane. In any other case, oscillations are excited and the tidal deformations which are time-dependent become inevitably dissipative. We will focus on the situation in which the stellar rotation period differs from the orbital period, that is, a non-synchronously rotating system. To describe the degree of non-synchronicity, we define $\beta=\omega_{0} / \Omega$, where $\omega_{0}$ and $\Omega$ are the rotational and orbital angular velocities, respectively.

As in the case of the terrestrial tides, the resulting asymmetrical shape of the star is due to a second order gravitational effect, the differential force. Thus, the rising tidal bulges, opposite to each other and comparable in size, are associated with the so called, equilibrium tide. Additionally, a set of surface oscillations commonly known as the dynamical tide, characterized by a wide variety of harmonic frequencies, is established. This oscillatory response of the star depends on $\beta$ and on the stellar and orbital parameters. In addition, it depends on the capability of the stellar material to transfer angular momentum and energy between its different layers, which is represented by the viscosity, $v$.

The tidally induced oscillations have both radial and azimuthal components. If we focus on the azimuthal component and keep in mind that the tidal forces decrease as $r^{3}$, with $r$ the distance between a mass element and the center of the perturbing star, it is evident that since an external layer of a perturbed star is subjected to a larger tidal force than a layer below it, a differentially rotating structure will be produced in any $\beta \neq 1$ binary system. If $v \neq 0$, the interaction between the different layers takes the form of a shearing flow, thereby leading to energy dissipation. The viscosity and the azimuthal velocity are the crucial parameters defining the amount of energy that is dissipated, $\dot{E}$.

The long term trend of the angular momentum transfer and dissipative effects in binary systems is one in which the rotation frequency becomes synchronous with the orbital frequency. The theoretical framework for the calculation of tidal synchronization was developed by Zahn (1966, 1977, 1989), who invoked the tidal friction as a physical mechanism asociated with tidally excited oscillations which contribute to the angular momentum transfer. In stars with convective envelopes, redistribution of angular momentum occurs through dissipation processes like 
turbulent viscosity, retarding the equilibrium tide. In stars with radiative envelopes, the mechanism found by Zahn $(1975,1977)$ is the action of radiative damping taking place on the dynamical tide. Zahn $(1977,1989)$ found the characteristic timescale for synchronization as a function of $a$, the major semi-axis of the orbit; $\tau_{\text {sync }} \sim a^{6}$ for stars with convective envelopes and $\tau_{\text {sync }} \sim$ $a^{8.5}$ for stars with radiative envelopes.

On orbital timescales, the tidal oscillations can produce observable photometric and absorption line-profile variability (see, for example, Smith 1977; Gies \& Kullavanijaya 1988; Willems \& Aerts 2002). Moreno \& Koenigsberger (1999) presented a simplified model for the calculation of the surface oscillations of a star driven by the tidal interaction in a binary system. The calculation is performed for a thin surface layer of small surface elements distributed along the equatorial belt of the star. The solution of the equations of motion for these surface elements provides the velocity perturbations in the radial and azimuthal directions as a function of time within the orbital cycle of the binary. In the non-inertial reference frame used to solve the equations of motion the model includes the gravitational forces of both stars, gas pressure, centrifugal, coriolis, and viscous forces. In the model we compute only the motion of the center of mass of each surface element, thus ignoring the details of the inner motions within these elements. In this approximation, the buoyancy force is not taken into account. Finally, we note that the results in this paper are obtained using a polytropic index $n=1.5$, corresponding to a rigorous convective envelope, instead of $n=1$ as was used previously (Moreno et al. 2005; Koenigsberger et al. 2006), although the difference between the results obtained for these two values of $n$ is not significant.

Moreno et al. (2005) extended the model to include the computation of the photospheric absorption lines that would be produced by the perturbed stellar surface. This ab initio calculation of the line profile variability due to the forced tidal oscillations was applied to the case of $\epsilon$ Per, showing that from a qualitative standpoint, the predicted line profile variability is comparable to the observations. More recently, the model was used to analyze the peculiar line-profile variability in the optical counterpart of the X-ray binary 2S0114+650 (Koenigsberger et al. 2006), leading to the conclusion that the tidal perturbations could be the "trigger" for the periodic ejection of wind material at densities that are higher than the average wind density. They hypothesize that these effects result from the combined action of the shear energy dissipation, the radial oscillations and the radiation pressure. The significance of this result is that, in addition to providing a tool for studying line profile variability in binary systems, the model provides a means of constraining the value of the viscosity, which is the parameter involved in the energy dissipation mechanisms. Thus, although the model is designed to study the variability in binary systems on orbital timescales, it is of interest to explore whether it yields results that are consistent with the long-term behavior, specifically, the tidal evolutionary theories. That is, using the value of viscosity and the angular velocity perturbations obtained from the calculation, an estimate of the rate of dissipated mechanical energy, $\dot{E}$, can be derived. The timescale over which $\dot{E}$ becomes negligible is a measure of the synchronization timescale of the binary system. Thus, the results of this one-layer calculation of the tidal oscillations may be compared with the predictions of tidal synchronization theory such as those of Zahn $(1977,1989)$ yielding, on the one hand, a test of the Moreno et al. (2005) model and, on the other, a constraint on the numerical values of the viscosity. These are two of the objectives of this paper.
The expression for the calculation of the viscous energy dissipation rate is derived in Sects. 2 and 3; in Sect. 4 we present the results of our model calculations for a $5 M_{\odot}+4 M_{\odot}$ binary system at different orbital separations and compare our predictions with the synchronization timescales of Zahn; and in Sect. 5 we discuss the implications of this result and present the conclusions.

\section{Viscous angular momentum transport in a shearing medium}

The approximate treatment of angular momentum transfer between the equatorial surface layer and the rigidly-rotating inner region of the star is made assuming that the equatorial external layer behaves as a shearing thin disk, in analogy with the dissipation mechanisms described for accretion disks (see for example, Frank et al. 1985; Lynden-Bell \& Pringle 1974). In the assumed differentially rotating medium, tangential stresses between adjacent layers are assumed to be the mechanism of transport of angular momentum. Included in these is the angular momentum transport due to magnetic torques, which has been shown to be the dominant mechanism in recent stellar model calculations (Petrovic et al. 2005) where the formulation of Spruit (2002) is used to treat the magnetic field. In our calculations, the mechanisms by which transfer of angular momentum in the shear flow occurs are englobed in the viscosity.

We adopt the approach for the transport of angular momentum that consists of imagining an idealized situation in which parts of a ring-shaped outer layer slide over a rigidly-rotating inner region. If friction between adjacent layers is assumed to exist and if the angular velocity increases or decreases outwards, a net torque will be exerted by the outer ring on the inner region. The resulting torques will work towards synchronizing the outer and inner region, dissipating energy in the process.

For this mechanism to be active, the particles in the external ring have to be able to interact with those in the inner rotating region. However, contrary to the case of many accretion disk models where the particle velocity is Keplerian, the velocity of different layers on a stellar surface is determined by the stellar rotation, the tidal forcing and the viscosity of the material.

Let us assume that the viscosity in the gaseous shearing medium is a turbulent one. Gas elements with small random motions at a typical turbulent velocity $v_{\text {turb }}$, travel a distance $\lambda_{\text {turb }}$ (the characteristic length and velocity scales of the turbulence), in all directions before mixing with the surroundings. There exists a typical turbulent viscosity,

$v \sim \lambda_{\text {turb }} v_{\text {turb }}$.

However, both $\lambda_{\text {turb }}$ and $v_{\text {turb }}$ are unknown parameters. A clever solution to this problem was proposed in the early 1970' s by Shakura \& Sunyaev (1973), consisting of a simple parametrization of the two turbulence scales. For the length scale, they assumed isotropy and characterized it by the typical size of the largest turbulent eddies which cannot exceed the thickness $H$ of the assumed ring $\lambda_{\text {turb }}<H$. For the velocity scale, a more straightforward scaling could be made. That is, if the turbulent velocity were supersonic, emerging shock waves would dissipate the energy and reduce the velocity to that of, or below, the sound speed. This leads to the choice of a $v_{\text {turb }}$ that is subsonic, thus $v_{\text {turb }}<C_{\mathrm{s}}$, and the viscosity can be written;

$v \sim \alpha H C_{\mathrm{s}}$

with $\alpha<1$. This is the well-known $\alpha$-prescription of Shakura $\&$ Sunyaev (1973). 
The physical idea of the viscous torque $g$ is to assume that, if the gas flow is turbulent to some extent, gas particles of adjacent layers will be exchanged in the radial direction (Frank 1985). Since the two different radial flows of material originating in both layers will have different specific angular momenta, this will cause a net transfer of angular momentum between them.

We can then calculate the magnitude of the viscous torque on the equatorial stellar region in terms of a kinematic turbulent viscosity $v$, which can be expressed as $v=\mu / \rho$. Here $\rho$ is the local density of the gas and $\mu$ is the coefficient of shear viscosity which is related to the component $\tau_{r \phi}$ of the viscous stress tensor in cylindrical coordinates $(r, \phi, z)$ by (Landau \& Lifshitz 1984):

$\tau_{r \phi}=\mu\left(\frac{1}{r} \frac{\partial u_{r}}{\partial \phi}+\frac{\partial u_{\phi}}{\partial r}-\frac{u_{\phi}}{r}\right)$

where $u_{r}, u_{\phi}$ are the radial and azimuthal components of the velocity field. Now, inserting $u_{\phi}=\omega_{\phi} r$ with $\omega_{\phi}$ as the rotational angular velocity of the tidal shear flow,

$\frac{\partial u_{\phi}}{\partial r}=\frac{\partial\left(r \omega_{\phi}\right)}{\partial r}=\omega_{\phi}+r \frac{\partial \omega_{\phi}}{\partial r}$,

and neglecting the shear effects in the radial direction, the viscous stress tensor becomes,

$\tau_{r \phi}=\mu r \frac{\partial \omega_{\phi}}{\partial r}$

This is a tangential force per unit area, and multiplying $\tau_{r \phi}$ by the lever $\operatorname{arm} r$, gives the viscous torque per unit area exerted by the sliding ring-shaped outer layer on the rigidly-rotating inner region. Integrating over the area of interaction $(\mathrm{d} A=r \mathrm{~d} \phi \mathrm{d} z)$,

$$
\begin{aligned}
g & =\int r \tau_{r \phi} \mathrm{d} A=\int \mu r^{3} \frac{\partial \omega_{\phi}}{\partial r} \mathrm{~d} \phi \mathrm{d} z \\
& =v \int_{0}^{2 \pi} r^{3} \frac{\partial \omega_{\phi}}{\partial r} \mathrm{~d} \phi \int_{-H / 2}^{H / 2} \rho \mathrm{d} z \\
& =v \Sigma \int_{0}^{2 \pi} r^{3} \frac{\partial \omega_{\phi}}{\partial r} \mathrm{~d} \phi
\end{aligned}
$$

where we have used that the density per unit area on the equatorial plane of the equatorial stellar region is

$$
\Sigma=\int_{-H / 2}^{H / 2} \rho \mathrm{d} z
$$

with $H$ the width of the equatorial stellar region. With $\Sigma=\rho H$, the torque can also be expressed as

$g=v \rho H \int_{0}^{2 \pi} r^{3} \frac{\partial \omega_{\phi}}{\partial r} \mathrm{~d} \phi$

According to this model, no net angular momentum is transported unless there is shearing differential rotation; that is, the condition for net angular momentum transport is, $\partial \omega_{\phi} / \partial r \neq 0$.

\section{The energy dissipation caused by the differential viscous torque}

A natural consequence of the shearing differential rotation is the dissipation of energy, $\dot{E}$. Let us consider the energy dissipation caused by the viscous torque acting on the stellar equatorial external layer between $r$ and $r+\Delta r$. The net differential viscous torque on this layer is

$g(r+\Delta r)-g(r) \sim \frac{\partial g}{\partial r} \Delta r$

Since the torque is acting in the direction of the angular velocity $\omega_{\phi}$, the rate of work exerted onto the external layer is;

$\omega_{\phi} \frac{\partial g}{\partial r} \Delta r=\frac{\partial}{\partial r}\left(g \omega_{\phi}\right) \Delta r-g \frac{\partial \omega_{\phi}}{\partial r} \Delta r$

where the first term of the right-hand side represents the rate of transported mechanical energy through the gas by the torque, and the term $-g\left(\partial \omega_{\phi} / \partial r\right) \Delta r$, the corresponding local rate of loss of mechanical energy converted into heat in the gas. We are interested in the dissipation caused by the viscous torques within the gas at a rate $g\left(\partial \omega_{\phi} / \partial r\right) \Delta r$ on the external layer of width $\Delta r$.

The local dissipation rate on an equatorial surface element with an azimuthal width $\Delta \phi$ is

$$
\begin{aligned}
\dot{E}_{\text {elem }} & =g \frac{\partial \omega_{\phi}}{\partial r} \Delta r=v \rho H r^{3}\left\{\frac{\partial \omega_{\phi}}{\partial r}\right\}^{2} \Delta r \Delta \phi \\
& =v \rho\left\{r \frac{\partial \omega_{\phi}}{\partial r}\right\}^{2} r \Delta \phi \Delta r H .
\end{aligned}
$$

Since we require an estimate of the dissipation rate on the entire surface of the star, the previous description must be extended to include the dissipation produced at polar angles $(\theta)$. An approximate generalization can be performed considering that the same dissipation mecanisms apply to a series of rings of thickness $r \Delta \theta$, situated across the star from the north pole to the south pole, and the elements in these rings with an azimuthal angle $\phi$ have the same angular velocity as that of the equatorial element with the same azimuth $\phi$. This approximation was used by Moreno et al. (2005) for the line-profile calculation.

From Eq. (14), the local dissipation rate on any surface element is

$\dot{E}_{\text {elem }}=v \rho\left\{r \frac{\partial \omega_{\phi}}{\partial r} \sin \theta\right\}^{2} r(\sin \theta) \Delta \phi \Delta r(r \Delta \theta)$

with $\omega_{\phi}$ the angular velocity of the equatorial surface element in the meridian of the element. Integrating in polar angle, we obtain the dissipation rate on a meridional shell,

$$
\begin{aligned}
\dot{E}_{\text {merid.shell }} & =v \rho\left\{r \frac{\partial \omega_{\phi}}{\partial r}\right\}^{2} r^{2} \Delta \phi \Delta r \int_{0}^{\pi} \sin ^{3} \theta \mathrm{d} \theta \\
& =\frac{4}{3} v \rho\left\{r \frac{\partial \omega_{\phi}}{\partial r}\right\}^{2} r^{2} \Delta \phi \Delta r
\end{aligned}
$$

To obtain the total dissipation rate $\dot{E}$, this equation must be integrated over the azimuthal coordinate $\phi$. We use the following approximation: $\left(\partial \omega_{\phi} / \partial r\right) \sim\left(\Delta \omega_{\phi} / \Delta r\right)_{i}$, where $\Delta \omega_{\phi} \sim \Delta V\left(\phi_{i}\right) / r_{i}$, with $r_{i}$ the radial position of the $i$ th equatorial surface element, and $\Delta V\left(\phi_{i}\right)$ the difference of azimuthal velocity of the element and the inner stellar region. Then, with $(\Delta r)_{i}$ the radial thickness 
of the element, the total energy dissipation can be obtained as a sum over the $n$ equatorial surface elements,

$$
\begin{aligned}
\dot{E} & \sim \frac{4}{3} v \rho \sum_{i}^{n}\left\{r^{2} \frac{\Delta \omega_{\phi}}{\Delta r}\right\}_{i}^{2} \Delta \phi_{i}(\Delta r)_{i} \\
& \sim \frac{4}{3} v \rho \sum_{i}^{n}\left\{\frac{r_{i} l_{i}}{(\Delta r)_{i}}\left(\Delta V\left(\phi_{i}\right)\right)^{2}\right\}
\end{aligned}
$$

where additionally, $r_{i} \Delta \phi_{i}$ is simplified by the factor $l_{i}$, which represents the azimuthal length of the element.

\section{Synchronization timescales}

For a super-synchronously rotating star, the transfer of angular momentum tends to brake the outer stellar layers, causing the total energy of the system to gradually decline. This, in turn, drives the system towards synchronization and, therefore, $\left|\Delta V\left(\phi_{i}\right)\right| \rightarrow 0$.

Zahn $(1977,1989)$ found two different relations between the synchronization timescale and the orbital separation in binary systems, depending on whether the star's outer envelope is radiative, $\tau_{\text {Zahn }} \sim\left(a / R_{1}\right)^{8.5}$, or convective, $\tau_{\text {Zahn }} \sim\left(a / R_{1}\right)^{6}$. He proposed that for stars with convective envelopes, the most efficient synchronizing mechanism is due to turbulent viscosity. In what follows, we will concentrate on this formulation, a choice that is motivated a posteriori by the results we derive and by the possibility that, even in stars with radiative envelopes, turbulence is induced by the differential rotation.

Zahn (1977) gives in his Eq. (6.1) an approximate characteristic synchronization timescale

$\tau_{\text {Zahn }} \sim q^{-2}\left(a / R_{1}\right)^{6} \sim 10^{4}((1+q) / 2 q)^{2} P^{4} \quad$ years

where $q=M_{2} / M_{1}$ is the mass ratio of the secondary to the primary star and $a$ is the orbital separation in solar units, $R_{1}$ is the equilibrium radius of the star in solar units and $P$ is the orbital period in days.

Adopting our one-layer model for calculating the tidal oscillations, and assuming that all of the energy dissipation associated with the shearing forces occurs on this surface layer, we can estimate the timescale required for the rotation period of the star to be synchronized with the orbital period of its companion as follows. If $E_{\text {rot }}^{\text {init }}$ and $E_{\text {rot }}^{\text {sync }}$ are the present and synchronization stellar rotational energies, computed with the moment of inertia of a rigid sphere and the azimuthal velocity at the stellar equator, $E_{\text {rot }} \sim(1 / 2) I(\omega)^{2} \sim(1 / 5) M_{1} V_{\text {rot }}^{2}$, then a rough estimate of the timescale needed to synchronize the system is

$\tau_{\mathrm{sync}} \sim \frac{\frac{1}{5} M_{1}\left(V_{\mathrm{rot}}^{\mathrm{init}}\right)^{2}-\frac{1}{5} M_{1}\left(V_{\mathrm{rot}}^{\mathrm{sync}}\right)^{2}}{\frac{4}{3} v \rho \sum_{i}^{n}\left\{\frac{r_{i} l_{i}}{(\Delta r)_{i}}\left(\Delta V\left(\phi_{i}\right)\right)^{2}\right\}}$.

\subsection{Comparison with Zahn's synchronization timescales}

In order to compare the above synchronization rates with those that are inferred from Zahn's $\left(a / R_{1}\right)^{6}$ prediction, our code was used to compute values of $\Delta V\left(\phi_{i}\right)$ and $r_{i}$ for a set of binary models with different orbital periods.

The test binary system that was selected consists of a Main Sequence intermediate-mass system with $M_{1}=5 M_{\odot}, M_{2}=$ $4 M_{\odot}$ and $R_{1}=3.2 R_{\odot}$ in a circular orbit. Observational evidence (Tassoul 2000; Pan 1997; Mathews \& Mathieu 1992;
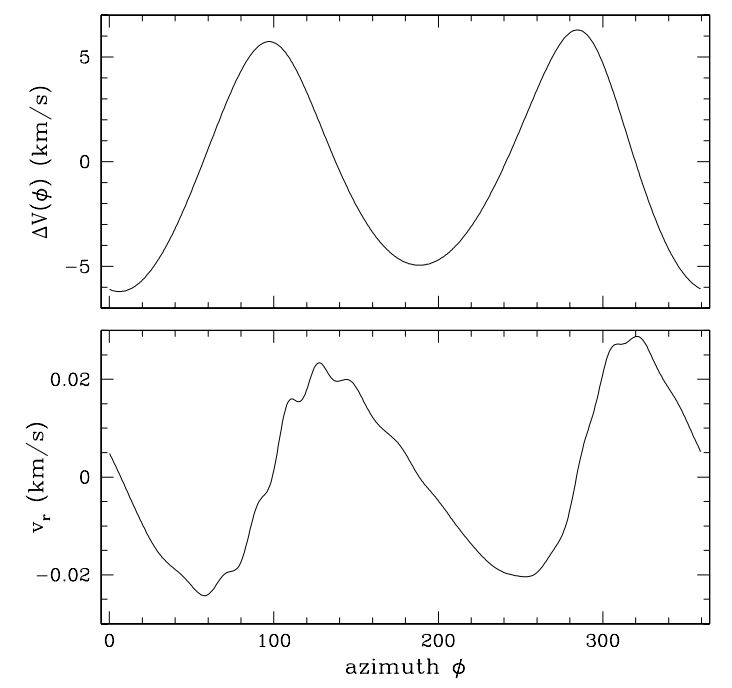

Fig. 1. Top: the difference of azimuthal velocity of each surface element and the rigidly rotating inner stellar body, $\Delta V(\phi)$ along the equatorial region for a $P_{\text {orb }}=5 \mathrm{~d}$ binary system. The angle $\phi$ is measured with respect to the line connecting the two stars. Bottom: the component of the velocity in the radial direction of each surface element along the equatorial belt for the same model. The star is rotating at twice the orbital frequency.

Witte \& Savonije 1999) provides constraints for the circularization timescales of such systems, and $\tau_{\text {sync }}$ is found to be shorter than $\tau_{\text {circ }}$ (Claret \& Cunha 1997; Zahn 1977; Hut 1981).

We chose to fix the value of $\beta=\omega_{0} / \Omega=2$, which means that for decreasing orbital separations, the rotational angular velocity, $\omega_{0}$, increases proportionately with the increasing orbital angular velocity $\Omega$. Although this might seem like a somewhat arbitrary choice, it is important to note that it is $\beta$ rather than $V_{\text {rot }}$ that plays a fundamental role in defining the frequencies and amplitudes of the oscillations. An intuitive way this can be understood is to consider two binary systems with different $\omega_{0}$, but both with $\beta=1$. Because $\beta=1$ corresponds to the equilibrium condition, no oscillations are present, regardless of how much larger one of the values of $\omega_{0}$ may be with respect to the other one.

Figure 1 illustrates the example of $\Delta V(\phi)$ and $V_{r}$ along the equatorial belt for the $P_{\text {orb }}=5$ day binary system. The abscissa corresponds to the location of the surface element along the equator with respect to the line connecting the two stars. The largest differences between the surface azimuthal velocity and the inner rigid-body rotating region are in general associated with the tidal bulges $\left(\phi \sim 0\right.$ and $\left.180^{\circ}\right)$ and the locations perpendicular to the axis of the orbit, and it is at these locations where the largest energy dissipation occurs. Note that the tangential component is $\sim 2$ orders of magnitude larger than the radial component, making it the dominant source of energy dissipation ${ }^{1}$. When the orbital separation decreases, the azimuthal variation is no longer smooth, with shorter scale oscillations appearing, as illustrated in Fig. 2 where we plot $\Delta V(\phi)$ for the $P_{\text {orb }}=2.5$ day binary system. Because we are considering circular orbits, $\Delta V(\phi)$ shown in Figs. 1 and 2 do not change significantly as a function of orbital phase and it is possible to assign a single $\Delta V(\phi)$ curve to each of the models. This would not be the case if the orbit were eccentric, where strong changes occur between periastron and apastron phases.

1 It is also the dominant source of photospheric absorption line profile variability as shown by Moreno et al. (2005). 


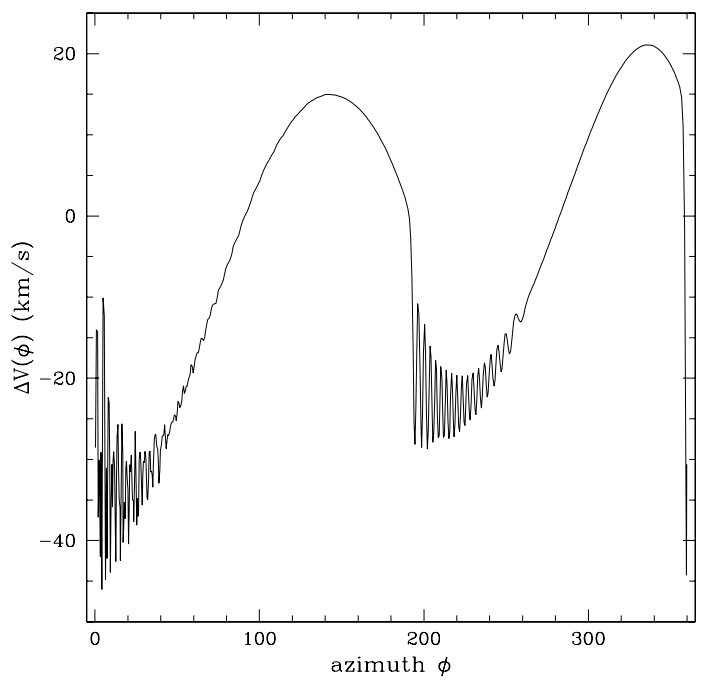

Fig. 2. The azimuthal velocity $\Delta V(\phi)$ along the equatorial region of surface elements with angle $\phi$ at a given time, for the $P_{\text {orb }}=2.5$ day binary system and with $\delta R=0.01 R_{1}$ and $v=0.0043 R_{\odot}^{2} \mathrm{~d}^{-1}$.

The value of the external oscillating layer's density, $\rho$, was obtained by computing stellar structure models using the code described in Yoon \& Langer (2005). The value $\rho=10^{-7} \mathrm{~g} \mathrm{~cm}^{-3}$ that we adopted corresponds to the average density in the external 73 layers of the theoretical $5 M_{\odot}$ star. These 73 layers comprise approximately $\delta R / R_{1}=0.01$, which is the thickness of the outer layer that we adopted for the oscillations calculation.

The remaining input parameter that needs to be defined for the oscillations code as well as for computing the synchronization times using Eq. (21) is the viscosity, $v$. Our practice (see Moreno et al. 2005) has been to use the smallest value of $v$ allowed by the code. That is, when the oscillation amplitudes are very large, the surface elements may either overlap with or become detached from their neighboring elements, at which time the computation can no longer proceed. This is prevented by assigning a sufficiently large value to the viscosity parameter. Our initial set of calculations were performed with $v=$ $0.005 R_{\odot}^{2}$ day $^{-1}$, which is slightly larger than the minimum $v$ allowed for the $P_{\text {orb }}=2.5$ day binary system. The results yielded a least-means-square fit with $\tau_{\text {sync }} \alpha\left(a / R_{1}\right)^{5.7}$, which is similar to that of $\tau_{\text {Zahn }} \alpha\left(a / R_{1}\right)^{6}$, but displaced vertically on the $\log \tau_{\text {sync }}$ vs. $a$ plane. Thus, the next step consisted in adjusting the values of $v$ to make $\tau_{\text {sync }}$ coincide with $\tau_{\text {Zahn }}$. The resulting values of $v$ and energy dissipation rates are listed in the last two columns of Table 1, and the synchronization timescales are plotted in Fig. 3. Note that the $0.0015-0.0043 R_{\odot}^{2} / \mathrm{d}$ range in $v$ suggests that part of the turbulent viscosity is itself produced through the tidal perturbations processes, since the closest binaries require the largest values of $v$.

We thus find that the shear energy dissipation due to the tangential velocity components of the surface layer used in our approximate treatment of the tidal interaction leads to synchronization timescales that are consistent with those predicted by Zahn's treatment of the star's entire structure for the case in which the outer envelope is convective.

\subsection{Dependence on $v$ and $\delta R / R_{1}$}

The viscosity and the thickness of the oscillating layer are free parameters that are not a priori constrained. We find that for a given orbital separation, the energy dissipation rates may span
Table 1. Binary system models for the $\tau_{\text {sync }}$ vs. $a$ calculation.

\begin{tabular}{cccccc}
\hline \hline $\begin{array}{c}P_{\text {orb }} \\
(\mathrm{d})\end{array}$ & $\begin{array}{c}a \\
\left(R_{\odot}\right)\end{array}$ & $\begin{array}{c}V_{\text {orb }} \\
\left(\mathrm{km} \mathrm{s}^{-1}\right)\end{array}$ & $\begin{array}{c}V_{\text {rot }} \\
\left(\mathrm{km} \mathrm{s}^{-1}\right)\end{array}$ & $\begin{array}{c}v \\
\left(R^{2} / \mathrm{d}\right)\end{array}$ & $\begin{array}{c}\dot{E} \\
\mathrm{erg} \mathrm{s}^{-1}\end{array}$ \\
\hline 2.5 & 16.11 & 326.26 & 130.00 & 0.0043 & $1.38 \times 10^{34}$ \\
5.0 & 25.58 & 258.96 & 64.80 & 0.0015 & $2.59 \times 10^{32}$ \\
7.5 & 33.52 & 226.22 & 43.20 & 0.0024 & $2.20 \times 10^{31}$ \\
10.0 & 40.60 & 205.53 & 32.40 & 0.0028 & $3.99 \times 10^{30}$ \\
12.5 & 47.12 & 190.80 & 25.90 & 0.0030 & $1.06 \times 10^{30}$ \\
15.0 & 53.21 & 179.55 & 21.60 & 0.0031 & $3.45 \times 10^{29}$ \\
17.5 & 58.97 & 170.55 & 18.50 & 0.0031 & $1.39 \times 10^{29}$ \\
20.0 & 64.46 & 163.13 & 16.20 & 0.0031 & $6.16 \times 10^{28}$ \\
22.5 & 69.72 & 156.85 & 14.40 & 0.0031 & $3.02 \times 10^{28}$ \\
25.0 & 74.80 & 151.44 & 13.00 & 0.0031 & $1.60 \times 10^{28}$ \\
\hline & & & & &
\end{tabular}

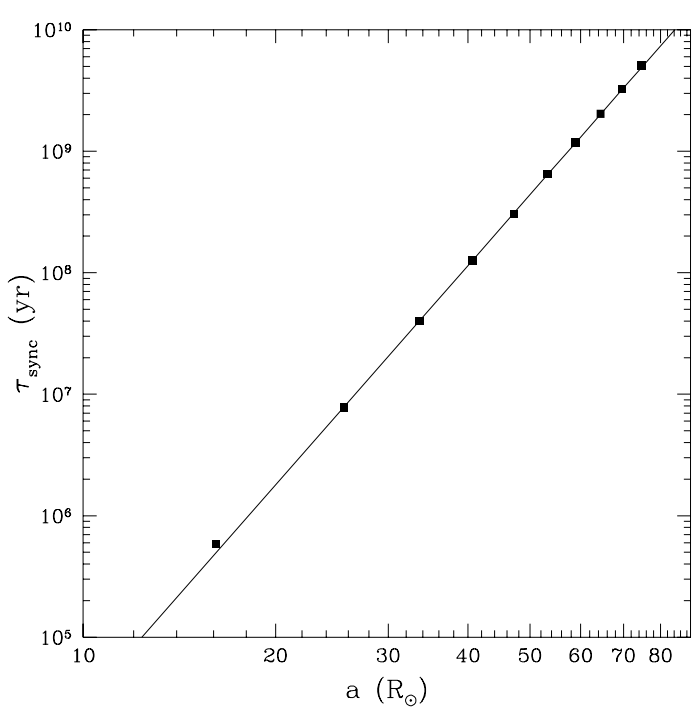

Fig. 3. Synchronization timescales (squares) calculated from our tidal oscillation model for a fixed $\beta=2$, and $\delta R=0.01 R_{1}$, and for values of $v$ as listed in Table 1 compared with Zahn's (1977) relation for stars with convective envelopes (continuous line).

a range of up to 2 orders of magnitude, depending on the combination of $v$ and $\delta R / R_{1}$. In Fig. 4 we illustrate the dependence of the energy dissipation rate on viscosity (left panels) and on the thickness of the layer (right panels). For $P_{\text {orb }}=2.5$ days, the energy dissipation rates decrease with increasing viscosity. Since the explicit dependence of $\dot{E}$ on the viscosity is linear, (see Eq. (17)) the energy dissipation would be expected to increase for increasing viscosity. On the other hand, because there is also a strong dependence of the dissipation rates on the amplitudes of the oscillations, and larger viscosities tend to reduce the amplitude of oscillation, the net result is a decrease in dissipation rates for larger viscosities. We thus see that for the closest binary systems, it is this decrease in oscillation amplitudes with increasing viscosities that dominates the behavior of $\dot{E}$.

For the longer orbital periods $\left(P_{\text {orb }}=10\right.$ days $)$, the interplay between the effect of increasing viscosity and decreasing oscillation amplitudes becomes more clear. In the middle panel of Fig. 4 we see how $\dot{E}$ first increases with increasing $v$ and then decreases as the oscillation amplitudes rapidly decline, due to the continued increase in $v$.

The thickness of the layer also has a strong influence on the value of $\dot{E}$. In the binary with the shortest orbital period, $\dot{E}$ increases systematically by up to 2 orders of magnitude when increasing $\delta R$ from $R_{1} / 200$ to $R_{1} / 50$. For longer period binaries, however (middle and bottom panels, right), there is a critical 

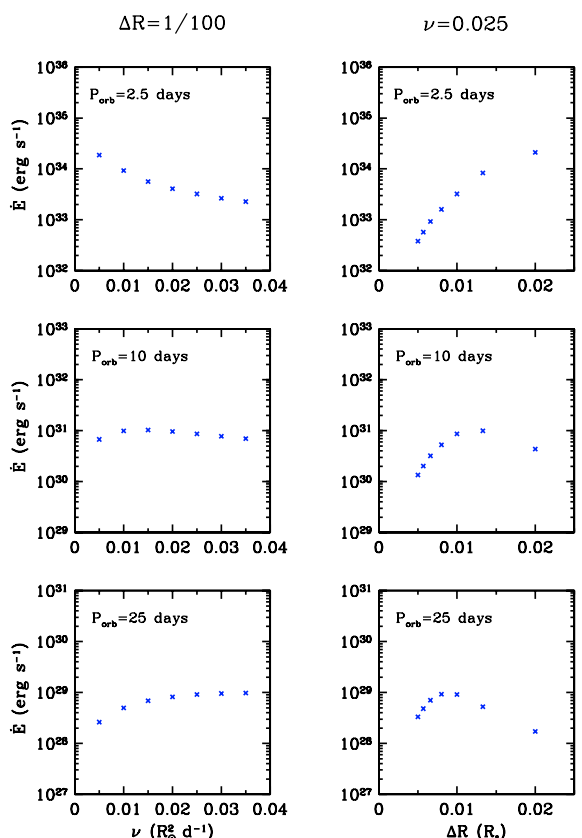

Fig. 4. Energy dissipation rates calculated for different viscosities $v$ with a fixed layer thickness $\delta R=0.01 R_{1}$ (left column) and for a changing layer thickness with a fixed viscosity $v=0.025 R_{\odot}^{2} \mathrm{~d}^{-1}$ (right column). Three binary systems with different orbital periods are illustrated, from top to bottom: $P_{\text {orb }}=2.5,10$ and $25 \mathrm{~d}$.

point at which $\dot{E}$ reaches a maximum value and then starts to decrease with increasing $\delta R / R_{1}$. For the $P_{\text {orb }}=10$ days system this critical value is $\delta R_{\mathrm{c}} \sim 1 / 75 R_{1}$ while for the $P_{\text {orb }}=25$ days case the turn around point appears at $\delta R_{\mathrm{c}} \sim 1 / 100 R_{1}$. Hence, the dependence of dissipation on the thickness of the layer is approximately linear up to the thickness $\delta R_{\mathrm{c}} / R_{1}$, with $\dot{E}$ increasing monotonically. However, once the layer is thicker than $\delta R_{\mathrm{c}} / R_{1}$, the surface layer has more inertia and its azimuthal velocity amplitudes become smaller and, once again, it is this effect that dominates the trend in $\dot{E}$.

\section{Discussion}

\subsection{The kinematical viscosity}

The energy dissipation rates computed from the results of the one-layer stellar oscillation model for binary systems lead to the possibility of constraining the value of the viscosity $v$. This can be performed by comparing the value of the synchronization timescale for a given binary system as obtained from our model $\tau_{\text {sync }}$, with the synchronization timescale curve predicted by Zahn's relation for the same system, $\tau_{Z \text { Zahn }}$. The difference between $\tau_{\text {sync }}$ and $\tau_{\text {Zahn }}$, if attributed to viscosity alone, allows its value to be determined. For the system parameters listed in Table 1 we derive $v$ in the range $0.0015-0.0043 R_{\odot}^{2} \mathrm{~d}^{-1}=0.84-2.41 \times 10^{14} \mathrm{~cm}^{2} \mathrm{~s}^{-1}$. If we apply the analogy with accretion disk studies where the viscosity is incorporated in the $\alpha$-parameter, an approximate relationship between kinematical viscosity and the Shakura \& Sunyaev $\alpha$ value is

$v \sim \alpha \frac{k T}{\mu m_{\mathrm{H}}} \frac{1}{\omega}$

from where

$\alpha \sim 0.0981 \frac{\left(\Delta V_{\phi} / \mathrm{km} \mathrm{s}^{-1}\right) \mu\left(v / R_{\odot}^{2} / \text { day }\right)}{\left(T /(10000 \mathrm{~K})\left(R_{1} / R_{\odot}\right)\right.}$.

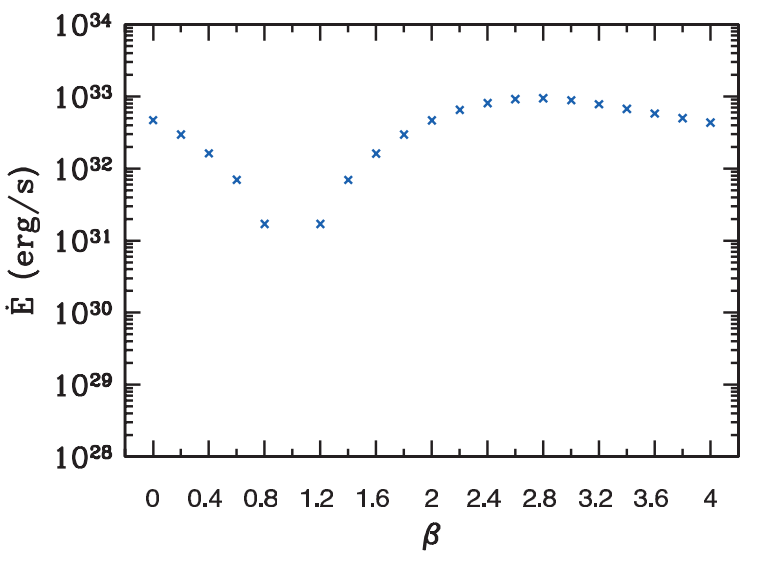

Fig. 5. Energy dissipation rates for different values of $\beta$, for a binary system with $M_{1}=5 M_{\odot}, M_{2}=4 M_{\odot}, R_{1}=3.2 R_{\odot}, P_{\text {orb }}=5 \mathrm{~d}, \delta R=$ $0.01 R_{1}$ and $v=0.005 R_{\odot}^{2} \mathrm{~d}^{-1}$.

Adopting the results illustrated in Fig. 2 for the maximum azimutal velocity, $\Delta V(\phi)$, assuming a mean molecular weight for a fully ionized gas, $\mu=0.62, T \sim 10000 \mathrm{~K}$, and using $v=$ $0.005 R_{\odot}^{2} \mathrm{~d}^{-1}$, the above equation yields values of $\alpha \leq 0.002$.

It is interesting also to compare this result with the values of the corresponding sum of diffusion coefficients for rotational mixing that are computed by the Binary Evolutionary Code (BEC; Petrovic et al. 2005, and references therein). In order to make this comparison, BEC was run for a $P_{\text {orb }}=5 \mathrm{~d}$ binary system with masses $5+4 M_{\odot}$. The computation was stopped after an evolutionary time of $\sim 10^{6}$ years in order to examine the values of these coefficients. The $\delta R=0.01 R_{1}$ layer that we adopted for the oscillations calculations is equivalent to 73 layers of the BEC computation, and the sum of the values of the combined diffusion coefficients for rotational mixing ${ }^{2}$ from these layers is $1.65 \times 10^{14} \mathrm{~cm}^{2} \mathrm{~s}^{-1}\left(=0.0030 R_{\odot}^{2} / \mathrm{d}\right)$. It is not, however, clear how the values obtained for these 73 layers should be combined in order to compare the result with the $v$ parameter that is used in the one-layer calculation, and the sum is most likely an overestimate. On the other hand, this value does not include the effects due to a magnetic field, which would tend to increase the value of the diffusion coefficient for each layer.

\subsection{Evolution of energy dissipation rates}

The energy dissipation rate, which is extracted from the rotational energy $E_{\text {rot }}$ of the star through the action of de-spinning, is calculated from the difference between its initial rotational energy and the energy at the time of synchronization divided by the synchronization timescale. Thus, this represents an average of the energy dissipation rate over the time it takes the star to reach synchronization. However, the rate of dissipation is expected to be a function of time, gradually vanishing as the system approaches synchronization. Indeed, Fig. 5 illustrates the dependence on $\beta$ of the energy dissipation rate due to azimuthal motions, showing that as $\beta \rightarrow 1, \dot{E} \rightarrow 0$. Figure 5 corresponds to the model binary system with $P_{\text {orb }}=5$ days, $v=0.005 R_{\odot}^{2}$ day $^{-1}$, and other parameters as used for Table 1.

Though we have not addressed the case of excentric binary systems in this paper, it is important to note that due to the dependence of angular velocity with orbital separation, $\beta$ is a function of orbital phase. This effect, combined with the variation in

\footnotetext{
${ }^{2}$ See Heger (1998) for a summary and description of the diffusion coefficients that are computed.
} 
orbital separation, leads to strongly varying energy dissipation rates over the orbital cycle. An exhaustive study of the available parameter space is beyond the scope of the present paper, but will be the objective of a forthcoming investigation. It is, however, worth noting that within the framework of Zahn's (1977) theory, the synchronization timescales are several orders of magnitude smaller than the circularization timescales. Hence, all binary systems should evolve to pseudo-synchronization (i.e., $a v-$ erage orbital angular velocity = rotational angular velocity) long before the orbit is circularized. Thus, large numbers of asynchronous binary systems in circular orbits are not expected to exist for stars on the Main Sequence.

\subsection{Non-synchronous rotation and surface activity}

An interesting consequence suggested by Fig. 5 is that nonsynchonous binary systems will tend rapidly towards values of $\beta$ near unity, but will live for a relatively longer time near the equilibrium value, but without having achieved it. Given the very large uncertainties in the observable parameters, primarily $V_{\text {rot }} \sin i$ and $R_{1}$, it is not easy to establish whether a particular binary system has actually achieved equilibrium. Thus, there may be a large number of non-synchronously rotating binary systems in which observable effects due to the tidal oscillations may be present. In particular, the peculiar X-ray emission that is observed in a small number of B-star binary systems in which there is evidence for non-synchronous rotation (Haro et al. 2003) may be a manifestation of tidally-induced activity. Although the detailed mechanism for converting tidal energy into X-ray emission is not known, we have previously speculated (Moreno et al. 2005; Koenigsberger et al. 2006) that the shear produced by the relative azimuthal motions of the outer stellar layers may lead to magnetic field generation near the stellar surface as well as mass ejection episodes. Thus, the X-ray emission could be associated with these processes. The possible connection between surface activity and tidal interactions has already been investigated in connection with the distribution of star spots in RS CVn-type binary systems (Holtzwarth \& Schüssler 2002).

Other observational manifestations of this activity may be "turbulence" above the expected value for the effective temperature of the star, and photometric and line profile variability. For example, the theoretical absorption line profiles that were computed for the optical counterpart of the X-ray binary system 2S0114+650 required a "macroturbulence" parameter of $37.5 \mathrm{~km} \mathrm{~s}^{-1}$ to match the observations (Koenigsberger et al. 2006), significantly larger than the thermal speeds expected in a B1-supergiant star. However, the problem of excessively large "macroturbulent" velocities is widely encountered among massive stars and, to our knowledge, there has been no systematic study made to determine whether the problem is more severe in binary stars than in single stars.

\section{Conclusions}

We have shown that a single-layer approximation for the calculation of tidal oscillations yields results that are consistent with the predictions of Zahn's $(1966,1977,1989)$ theory for the synchronization timescales in circular orbits, $\tau_{\text {sync }} \sim a^{6}$, thus providing a simplified means of computing the energy dissipation rates, $\dot{E}$ in binary systems. Furthermore, by calibrating our model results to fit Zahn's relationship, we are able to constrain the value of the kinematical viscosity parameter, $v$. For the binary system models considered in this investigation, $v \sim 0.0015-0.0043 R_{\odot}^{2} /$ day.
This is significantly larger than values typically obtained from detailed computations of the interior structure of stellar convective layers, in the absence of magnetic fields.

Our code makes no assumption regarding the energy dissipation mechanism except that the dissipation processes can be described through the parameter $v$. We show in this paper that if we assume that the energy dissipation mechanism is related to turbulent viscosity, we arrive at Eq. (19), which allows us to compute energy dissipation rates using the output of the code, and we show that the derived energy dissipation rates are consistent with those predicted by theoretical models of stars with convective envelopes. However, the code includes no a priori assumptions regarding the energy transport mechanism. In order to compare the results of the code with the theory for stars having outer radiative envelopes, the effects due to radiative damping need to be incorporated in the derivation of the synchronization timescale, which goes beyond the scope of the present investigation.

In summary, however, we have shown that the Moreno et al. (2005) one-layer model yields results that are consistent with other theoretical calculations of the tidal interactions in binary systems. It has the advantage that it can be used to compute the tidal oscillations for arbitrary stellar rotational velocities and orbital excentricities, allowing an estimate for the numerical value of $v$ under general conditions in binary systems. In addition, the code provides values of the radial and tangential components of the oscillation velocities at any time throughout the orbit, as well as the location on the stellar surface where the largest shear instabilities may be occurring. This allows a comparison with observational quantities, such as absorption line profiles and diagnostics of surface activity and should allow a methodical exploration of the impact that tidal oscillations may have on the surface properties of stars in non-synchronously rotating binary systems.

Acknowledgements. GK thanks S $\phi$ ren Meibom, Luis Mochan and Daniel Proga for helpful discussions. Support from CONACYT grant 36569 and UNAM/PAPIIT IN119205 are acknowledged.

\section{References}

Claret, A., \& Cunha, N. C. S. 1997, A\&A, 318, 187

Frank, J., King, A. R., \& Raine, D. J. 1985, Accretion Power in Astrophysics (Cambridge University Press), 65

Gies, D. R., \& Kullavanijaya, A. 1988, ApJ, 326, 813

Haro, S., Juárez, J. J., \& Koenigsberger, G. 2004, in IAU Symp. 215, Stellar Rotation, ASPCS, ed. A. Maeder, \& P. Eenens, 163

Heger, A. 1998, Ph.D. Dissertation, Technische Universität, München, Fakultät für Physik, MPA 1120

Holzwarth, V., \& Schüssler, M. 2002, AN, 323, 399

Hut, P. 1981, A\&A, 99, 126

Koenigsberger, G., Georgiev, L., Moreno, E., et al. 2006, A\&A, 458, 513

Landau, L. D., \& Lifshitz, E. M. 1984, Fluid Mechanics (Pergamon Press)

Lynden-Bell, \& Pringle 1974, MNRAS, 168, 803

Mathews, L. D., \& Mathieu, R. D. 1992, ASP Conf. Ser., 32, 244 ed. H. McAlister, \& W. I. Hartkopf

Moreno, E., \& Koenigsberger, G. 1999, RMA\&A, 35, 157

Moreno, E., Koenigsberger, G., \& Toledano, O. 2005, A\&A, 437, 641

Pan, K. 1997, A\&A, 321, 597

Petrovic, J., Langer, N., Yoon, S.-C., \& Heger, A. 2005, A\&A, 435, 247

Shakura, N. I., \& Sunyaev, R. A. 1973, A\&A, 24, 337

Smith, M. A. 1977, ApJ, 215, 574

Spruit, H. C. 2002, A\&A, 381, 923

Tassoul, J.-L. 2000, Stellar Rotation (Cambridge University Press), ISBN 0521772184

Willems, B., \& Aerts, C. 2002, A\&A, 384, 441

Witte, M. G., \& Savonije, G. J. 1999, A\&A, 350, 129

Yoon, \& Langer, N. 2005, A\&A, 443, 643

Zahn, J. P. 1966, Annales d'Astrophysique, 29(4)

Zahn, J. P. 1975, A\&A, 41, 329

Zahn, J. P. 1977, A\&A, 57, 383

Zahn, J. P. 1989, A\&A, 220, 112 